\title{
Shape of Cooper pairs in a normal-metal/superconductor junction
}

\author{
Yukio Tanaka, ${ }^{1,2}$ Yasuhiro Asano ${ }^{3}$ and Alexander A. Golubov ${ }^{4}$ \\ ${ }^{1}$ Department of Applied Physics, Nagoya University, Nagoya 464-8603, Japan \\ ${ }^{2}$ CREST, Japan Science and Technology Cooperation (JST), Nagoya 464-8603 Japan \\ ${ }^{3}$ Department of Applied Physics, Hokkaido University, Sapporo 060-8628, Japan \\ ${ }^{4}$ Faculty of Science and Technology and Mesa+Institute for Nanotechnology, University of Twente, Enschede 7500AE, The Netherlands
}

(Received 10 April 2008; published 12 June 2008)

\begin{abstract}
In $s$-wave superconductors the Cooper pair wave function is isotropic in momentum space. This property may also be expected for Cooper pairs entering a normal metal from a superconductor due to the proximity effect. We show, however, that such a deduction is incorrect and the pairing function in a normal metal is surprisingly anisotropic because of quasiparticle interference. We calculate angle-resolved quasiparticle density of states in NS bilayers which reflects such anisotropic shape of the pairing function. We also propose a magnetotunneling spectroscopy experiment which could confirm our predictions.
\end{abstract}

DOI: 10.1103/PhysRevB.77.220504

PACS number(s): 74.45.+c, 74.50.+r, 74.20.Rp

It is well known that Cooper pairs consisting of two electrons are characterized by electric charge $2 e$, macroscopic phase, internal spin, and by time and orbital structures. ${ }^{1}$ The charge $2 e$ manifests itself in various experiments, like Shapiro steps, flux quantization and excess current due to the Andreev reflection. The macroscopic phase generates the Josephson current. ${ }^{1}$ The internal spin structure is classified into spin-triplet and spin-singlet states. Further, based on a symmetry with respect to the internal time, superconducting state can belong to the even-frequency or the odd-frequency symmetry class. ${ }^{2}$ The orbital degree of freedom is described by an angular momentum quantum number $l .^{3,4}$ The well established properties listed above hold in bulk superconductors. The presence of perturbations like spin-flip or interface scattering may change the symmetry of Cooper pairs. For instance, an unusual odd-frequency property of Cooper pairs in proximity structures was predicted in recent studies. 5,6 The shape of Cooper pair wave function in nonuniform systems like superconducting junctions is not necessarily the same as that in the bulk state. Despite the extensive study of the proximity effect in several past decades, rather little attention has been paid to the problem of Cooper pair shape in nonuniform superconducting systems. ${ }^{7,8}$ This issue is quite important in view of current interest in the physics of superconducting nanostructures.

The aim of the present Rapid Communication is to clarify the consequences of breakdown of translational symmetry in superconductors on the Cooper pair shape. For this purpose, we study the proximity effect in quasi-two-dimensional normal metal / superconductor (N/S) junctions by solving the Eilenberger equation. We analyze the pairing function and the local density of states (LDOS) in N/S junctions with spin-singlet $s$-wave and $d_{x y}$-wave superconductors. The shape of the Cooper pair deviates seriously from that of the bulk with the generation of the odd-frequency component of the pairing function due to the formation of the AndreevSaint-James bound states. ${ }^{9}$ To detect the complex Cooper pair shape, we propose to use scanning tunneling spectroscopy in rotating magnetic field. We show that the calculated tunneling conductance exhibits complex patterns even in the $s$-wave case.

Let us consider a quasi-two-dimensional N/S junction as shown in Fig. 1 which is the simplest example of a nonuniform superconducting system, where the $\mathrm{S}$ region is semiinfinite and the normal metal has finite length $L$. We consider a perfect N/S interface with perfect transmissivity, while it can be shown that characteristic behavior of Cooper pairs remains qualitatively unchanged even in the presence of a potential barrier at the N/S interface.

The quasiclassical Green's functions ${ }^{10}$ in a normal metal $(\mathrm{N})$ and a superconductor $(\mathrm{S})$ are parameterized as

$$
\hat{g}_{ \pm}^{(i)}=f_{1 \pm}^{(i)} \hat{\tau}_{1}+f_{2 \pm}^{(i)} \hat{\tau}_{2}+g_{ \pm}^{(i)} \hat{\tau}_{3}, \quad\left(\hat{g}_{ \pm}^{(i)}\right)^{2}=\hat{1}
$$

where a superscript $i(=N, S)$ refers to $\mathrm{N}$ and $\mathrm{S}, \hat{\tau}_{j}(j=1-3)$ are the Pauli matrices, and $\hat{1}$ is a unit matrix. The subscript + (-) denotes a moving direction of a quasiparticle in the $x$ direction, ${ }^{10}$ and $\bar{\Delta}_{+}(x)\left[\bar{\Delta}_{-}(x)\right]$ is the pair potential for a left (right) going quasiparticle. In a normal metal, $\bar{\Delta}_{ \pm}(x)$ is set to zero because the pairing interaction is absent there. The Green's functions can be expressed in terms of the Ricatti parameters, ${ }^{11}$

$$
\begin{gathered}
f_{1 \pm}^{(i)}=\mp \nu_{i}\left[\Gamma_{ \pm}^{(i)}(x)+\zeta_{ \pm}^{(i)}(x)\right] /\left[1+\Gamma_{ \pm}^{(i)}(x) \zeta_{ \pm}^{(i)}(x)\right], \\
f_{2 \pm}^{(i)}=i\left[\Gamma_{ \pm}^{(i)}(x)-\zeta_{ \pm}^{(i)}(x)\right] /\left[1+\Gamma_{ \pm}^{(i)}(x) \zeta_{ \pm}^{(i)}(x)\right]
\end{gathered}
$$

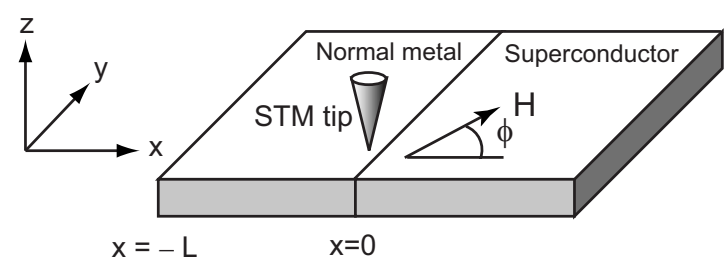

FIG. 1. A schematic of a N/S junction. A normal metal $(-L$ $<x<0)$ is attached to a superconductor $(0<x<\infty)$. To detect the deformation of a Cooper pair, we propose a STS experiment in the presence of magnetic field $(H)$ whose direction is perpendicular to the $z$ axis and oriented by $\phi$ from the $x$ axis. 


$$
g_{ \pm}^{(i)}=\left[1-\Gamma_{ \pm}^{(i)}(x) \zeta_{ \pm}^{(i)}(x)\right] /\left[1+\Gamma_{ \pm}^{(i)}(x) \zeta_{ \pm}^{(i)}(x)\right],
$$

with $\nu_{i}=1$ for $i=S$ and $\nu_{i}=-1$ for $i=N$. The parameters $\Gamma_{ \pm}^{(i)}(x)$ and $\zeta_{ \pm}^{(i)}(x)$ obey the Eilenberger equation of the Ricatti type, ${ }^{11}$

$$
\begin{gathered}
i v_{F x} \partial_{x} \Gamma_{ \pm}^{(i)}(x)=-\bar{\Delta}_{ \pm}(x)\left\{1+\left[\Gamma_{ \pm}^{(i)}(x)\right]^{2}\right\}+2 \bar{\varepsilon} \nu_{i} \Gamma_{ \pm}^{(i)}(x), \\
i v_{F x} \partial_{x} \zeta_{ \pm}^{(i)}(x)=-\bar{\Delta}_{ \pm}(x)\left\{1+\left[\zeta_{ \pm}^{(i)}(x)\right]^{2}\right\}-2 \bar{\varepsilon} \nu_{i} \zeta_{ \pm}^{(i)}(x),
\end{gathered}
$$

with $\bar{\varepsilon}=\varepsilon+i \delta_{0}$, where $\varepsilon$ is the energy of a quasiparticle measured from the Fermi level and $\delta_{0}$ is the level broadening due to impurity scattering. $v_{F x}$ is the $x$-component of Fermi velocity. In the clean limit, we consider $\delta_{0} \ll \Delta_{0}$. Boundary condition at $x=-L$ is given by $\zeta_{ \pm}^{(N)}(-L)=-\Gamma_{\mp}^{(N)}(-L)$. Boundary condition at the N/S interface becomes $\zeta_{ \pm}^{(S)}(0)=-\Gamma_{ \pm}^{(N)}(0)$ and $\zeta_{ \pm}^{(N)}(0)=-\Gamma_{ \pm}^{(S)}(0)$. The pair potential $\bar{\Delta}_{ \pm}(x)$ is expressed by $\bar{\Delta}_{ \pm}(x)=\Delta(x) \Phi_{ \pm}(\theta) \Theta(x)$, where a form factor $\Phi_{ \pm}(\theta)$ is given by $\Phi_{ \pm}(\theta)=1$ for $s$-wave symmetry and $\pm \sin 2 \theta$ for $d_{x y}$-wave one with $\theta$ being an incident angle of a quasiparticle measured from the $x$ direction. Bulk pair potential is $\Delta(\infty)=\Delta_{0}$, and we determine the spatial dependence $\Delta(x)$ in a selfconsistent way.

For $x \gg L_{0}$, the angular structure of $f_{2 \pm}^{(S)}$ follows that of the pair potential, whereas $f_{1 \pm}^{(S)}$ is zero with $L_{0}=v_{F} / T_{C}$ being a coherence length in $\mathrm{N}$ and $T_{C}$ being the transition temperature. The pairing function $f_{1 \pm}^{(i)}$ is generated by inhomogeneity in a system and thus has a finite value only near the interface and in a normal metal. Recent studies ${ }^{6}$ have shown that $f_{1+}^{(i)}$ has an odd-frequency symmetry since functions $f_{1 \pm}^{(i)}$ and $f_{2 \pm}^{(i)}$ have opposite parities. The induced odd-frequency component has the odd (even) parity, respectively. Pairing function $f_{1}$ is defined in the angular domain of $-\pi / 2 \leq \theta<3 \pi / 2$. We denote $f_{1}(\theta)$ by $f_{1+}(\theta)$ in the angle range $-\pi / 2 \leq \theta<\pi / 2$ and $f_{1}(\theta)=f_{1-}(\pi-\theta)$ for $\pi / 2 \leq \theta<3 \pi / 2$. The angular structure of functions $f_{2}$ and $g$ is defined in the same manner. LDOS is given by the relation $\rho_{L}(\theta)=\operatorname{Real}[g(\theta)]$. In what follows, we fix temperature $T=0.05 T_{C}$, the length of the normal region $L=5 L_{0}$, and $\delta_{0}=0.01 \Delta_{0}$.

In Figs. 2 and 3 we show polar plots of $f_{1}, f_{2}$, and $\rho_{L}$ in $s$-wave and $d_{x y}$-wave junctions for various choices of $\varepsilon$ and $x$. Dashed, solid and dotted lines represent, respectively, the results for $x=\infty$ (superconductor), $x=0$ (interface), and $x=-L / 2$ (normal metal). The odd-frequency component is always absent for $x=\infty$. Since $\rho_{L}$ is independent of $x$ in $\mathrm{N}$, the resulting value of $\rho_{L}$ at $x=0$ is equal to that at $x=-L / 2$.

First, we focus on the $s$-wave case (Fig. 2). As shown in Fig. 2(a), at $\varepsilon=0$ and $x=\infty, 0$ the even-frequency component $f_{2}$ has a circular shape reflecting the $s$-wave symmetry. However, at $x=-L / 2$, the shape is no longer a simple circular one but has a form of a doubly distorted circle. The shape of $f_{2}$ in a superconductor always has the circular shape independently of $\varepsilon$ as shown with dashed lines in Figs. 2(a), 2(d), and $2(\mathrm{~g})$. At $\varepsilon=0.1 \Delta_{0}$ in Fig. 2(d), $f_{2}$ at the interface (solid line) slightly deviates from the circular shape, while the shape in $\mathrm{N}$ drastically changes. The tendency is more remarkable at $\varepsilon=0.5 \Delta_{0}$ as shown in Fig. $2(\mathrm{~g})$. The butterflylike pattern of $f_{2}$ at the interface [solid line in Fig. $\left.2(\mathrm{~g})\right]$ is com-

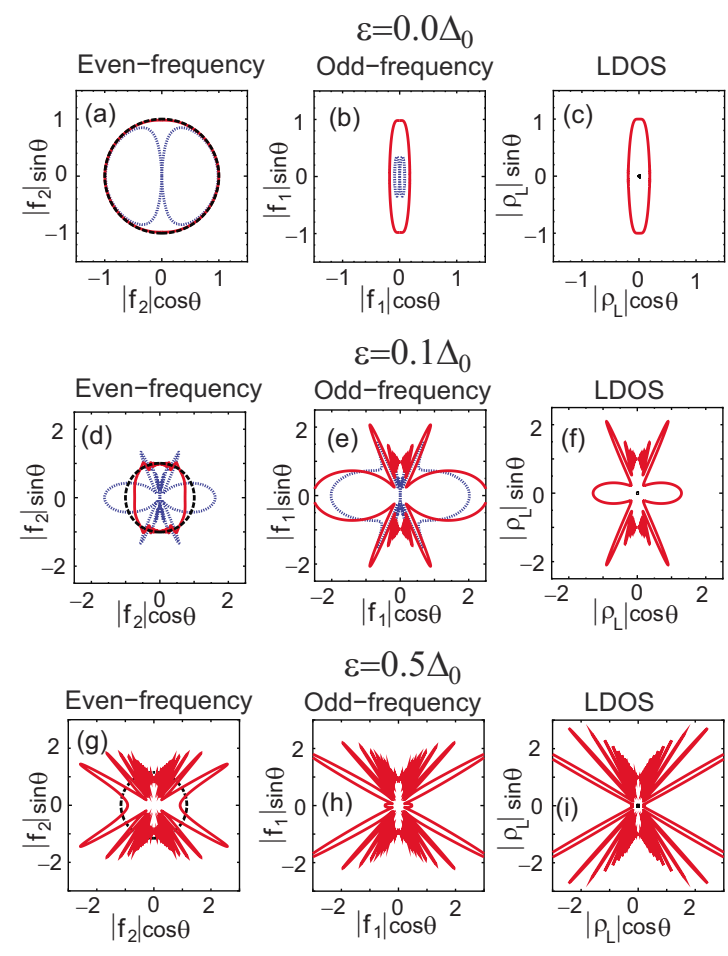

FIG. 2. (Color online) The results for s-wave symmetry. The shape of the even-frequency pair amplitude $[(\mathrm{a}),(\mathrm{d}),(\mathrm{g})]$, the oddfrequency one $[(\mathrm{b}),(\mathrm{e}),(\mathrm{h})]$, and the angle-resolved local density of states [(c), (f), (i)]. Solid lines: $x=0$ (at N/S interface), dotted lines: $x=-L / 2$ (in a normal metal) and dashed lines: $x=\infty$ (in a superconductor). $\varepsilon=0$ for (a), (b) and (c), $\varepsilon=0.1 \Delta_{0}$ for (d), (e) and (f), and $\varepsilon=0.5 \Delta_{0}$ for $(\mathrm{g}),(\mathrm{h})$ and (i). The angle $\theta$ is measured from the $x$ axis.

pletely different from the original circular shape in a superconductor. At $\varepsilon=0$, function $f_{1}$ at the interface becomes ellipsoidal as shown by the solid line in Fig. 2(b). The shape of $f_{1}$ at $x=0$ and $x=-L / 2$ exhibits the butterflylike pattern [Fig. $2(\mathrm{e})]$. For $\varepsilon=0.5 \Delta_{0}$, the line shape of $f_{1}$ has many spikes as shown in Fig. 2(h). Such anisotropic property of $f_{1}$ and $f_{2}$ affects the LDOS as shown in Figs. 2(c), 2(f), and 2(i). In particular, LDOS at the interface for $\varepsilon=0.5 \Delta_{0}$ [solid line in (i)] strongly deviates from the circular shape. At the interface, the shape of $\rho_{L}$ in Fig. 2(c) is quite similar to that of $f_{1}$ shown in Fig. 2(b).

These profiles can be qualitatively understood as follows. At $x=0$, the relations $f_{1 \pm}^{(N)}= \pm \Gamma\left(1-\alpha^{2}\right) / \Xi$, and $g_{ \pm}^{(N)}=(1$ $\left.+\alpha^{2} \Gamma^{2}\right) / \Xi$ are satisfied with $\Xi=1-\Gamma^{2} \alpha^{2}, \Gamma=\Gamma_{ \pm}^{(S)}(0)$ and $\alpha$ $=\exp \left[2 i \varepsilon L /\left(v_{F} \cos \theta\right)\right]$. For $\varepsilon \ll \Delta_{0}$, the relations $\Gamma \sim 1 / i$ and $f_{1 \pm} \sim i g_{ \pm}$are satisfied. Thus shape of function $f_{1}$ is similar to that of $\rho_{L}$. This argument is valid even for $\varepsilon=0.1 \Delta_{0}$ in Figs. 2(e) and 2(f), and for $\varepsilon=0.5 \Delta_{0}$ in Figs. 2(h) and 2(i). The oscillating behavior in $f_{1}, f_{2}$, and $\rho_{L}$ is more remarkable at $\varepsilon=0.5 \Delta_{0}$. Although we do not present calculated results of $f_{1}$ and $f_{2}$ at $x=-L / 2$ for $\varepsilon=0.5 \Delta_{0}$, the butterflylike pattern with many spikes in the pairing functions can be seen also in a normal metal. The directions of the spin projections in LDOS are characterized by small value of $\Xi$, which has close relation to the formation of the Andreev-Saint-James bound states. $^{9,12}$ For $\theta \sim \pm \pi / 2, \alpha$ oscillates rapidly with small 

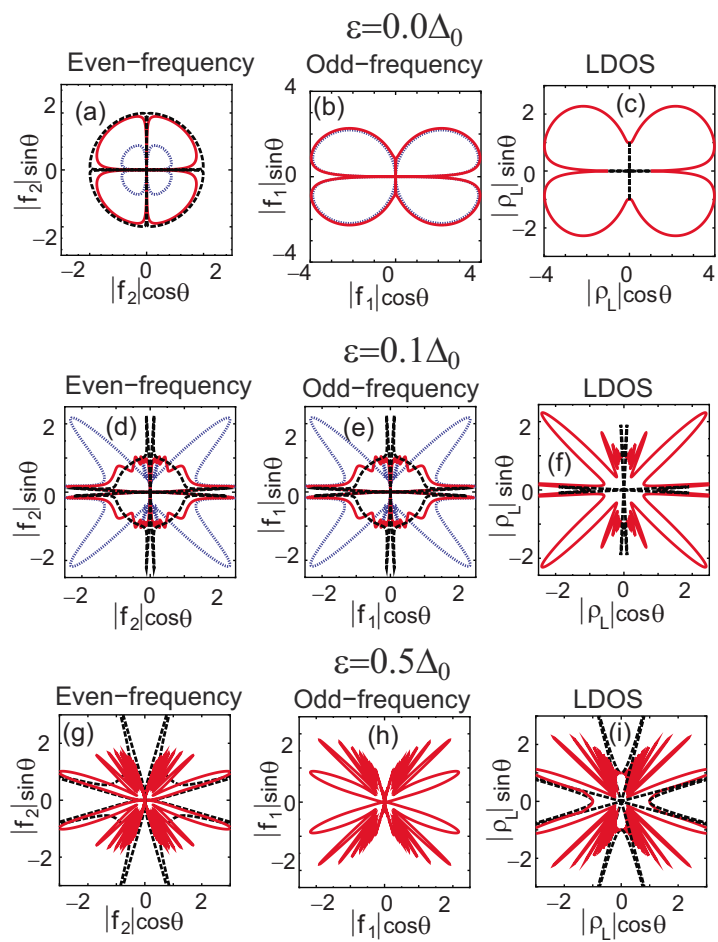

FIG. 3. (Color online) The results for $d_{x y}$-wave symmetry. The notations are the same as in Fig. 2.

variation of $\theta$, which explains the fine structures in LDOS around $\theta= \pm \pi / 2$. The quasiparticle interference effect is a source of Andreev-Saint-James bound state formation in a normal metal. As a result, the circular shape of Cooper pairs in $s$-wave superconductor is modified into the butterflylike pattern in a normal metal.

Next, we discuss the results for $d_{x y}$-wave junctions shown in Fig. 3. In a superconductor $(x=\infty)$, functions $f_{2}$ and $g$ are given by $\Delta_{0} \sin 2 \theta / \sqrt{\varepsilon^{2}-\Delta_{0}^{2} \sin ^{2} 2 \theta}$ and $\varepsilon / \sqrt{\varepsilon^{2}-\Delta_{0}^{2} \sin ^{2} 2 \theta}$, respectively. As shown by dashed lines in Figs. 3(d), 3(f), $3(\mathrm{~g})$, and 3(i), the amplitudes of $f_{2}$ and $g$ become large along the directions $\theta=\left[\sin ^{-1}\left(\varepsilon / \Delta_{0}\right)\right] / 2$. At $\varepsilon=0$ and $x=0$, formation of a mid-gap Andreev resonant state ${ }^{13}$ significantly enhances the amplitudes of $f_{1}$ and $\rho_{L}$ compared to that of $f_{2}$ as shown by solid lines in Figs. 3(a)-3(c). For $\varepsilon=0$ and $\varepsilon$ $=0.1 \Delta_{0}$, the shapes of $f_{1}$ and $\rho_{L}$ at the N/S interface are similar to those in $s$-wave superconductor junctions [solid lines in Figs. 3(b), 3(c), 3(e), and 3(f)]. At $\varepsilon=0.5 \Delta_{0}$, similar to the $s$-wave case, functions $f_{1}$ and $f_{2}$ in $\mathrm{N}$ have complex line shapes with many spikes.

Here, we propose an experimental setup to measure the complex Cooper pair shape, based on magnetotunneling spectroscopy, i.e., scanning tunneling spectroscopy (STS) in the presence of magnetic field. As shown in Fig. 1, magnetic field is applied parallel to the N/S plane. Tunneling current at a fixed bias voltage is measured as a function of the angle $\phi$ between the $x$ axis and the direction of magnetic field. The vector potential in this configuration is given by $\left(A_{x}, A_{y}\right)=$ $-\lambda H \exp (-z / \lambda)(\sin \phi, \cos \phi) .{ }^{14}$ We assume that thickness of a quasi-two-dimensional superconductor is sufficiently small compared to a magnetic field penetration depth $\lambda$. Magnetic field shifts the quasiparticle energy $\varepsilon$ to $\varepsilon-H \Delta_{0} \sin (\phi-\theta) /$
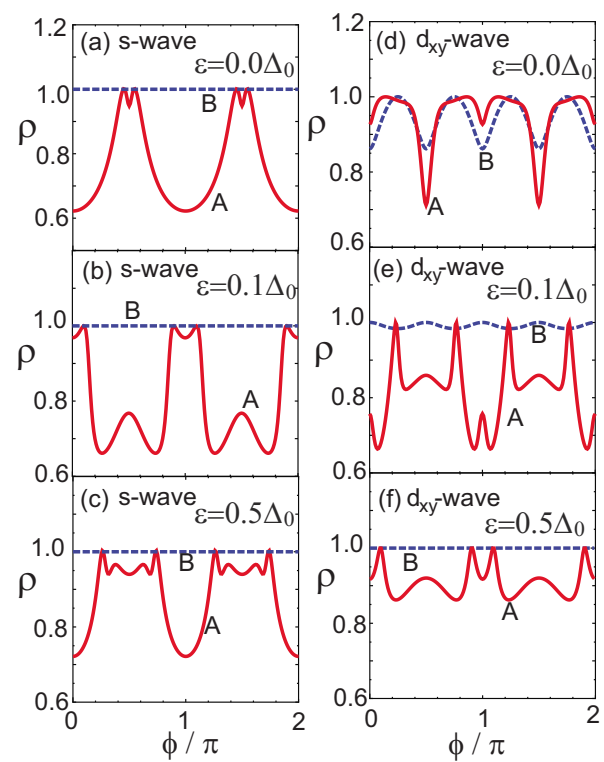

FIG. 4. (Color online) Normalized local density of states obtained in magnetotunneling spectroscopy as a function of the orientation angle of magnetic field $\phi$ in a spin-singlet $s$-wave junction $\left[(\mathrm{a}),(\mathrm{b})\right.$, and (c)] and in a spin-singlet $d_{x y}$-wave one $[(\mathrm{d}),(\mathrm{e})$, and (f)]. $\varepsilon=0$ for (a) and (d), $\varepsilon=0.1 \Delta_{0}$ for (b) and (e), and $\varepsilon=0.5 \Delta_{0}$ for (c) and (f). A: $x=0$ (at N/S interface) for $H=0.1 B_{0}$, and B: $x=\infty$ (in a superconductor) for $H=0.1 B_{0}$.

$B_{0}$, where $B_{0}=h /\left(2 e \pi^{2} \xi \lambda\right)$ and $\xi=\hbar v_{F} / \pi \Delta_{0}$. Here, to evaluate the order of magnitude of $B_{0}$, we explicitly write the Plank constant. For typical values of $\xi \sim \lambda \sim 100 \mathrm{~nm}$, the magnitude of $B_{0}$ is of the order of 0.02 Tesla. Local density of states measured in the considered magnetotunneling STS configuration is given by ${ }^{15} \rho_{S}(\phi)=\int_{-\pi / 2}^{3 \pi / 2} \rho_{L}(\theta, \phi) d \theta$. At sufficiently low temperatures the applied bias voltage $V$ satisfies the relation $e V=\varepsilon . \rho_{S}(\phi)$ is a periodic function of $\phi$ and we denote its maximum value as $\rho_{M}$. In the following, we focus on the normalized value $\rho(\phi)$ defined by $\rho(\phi)=\rho_{S}(\phi) / \rho_{M}$.

In Fig. 4, $\rho=\rho(\phi)$ is plotted as a function of $\phi$. In the $s$-wave case, $\rho(\phi)$ in bulk is always unity due to the isotropic nature of the $s$-wave pairing as shown by curve B. On the other hand, $\rho$ at the N/S interface has an oscillatory dependence due to the deviation of the Cooper pair shape from the circular one. It is remarkable that the line shape of curve A changes drastically with the increase in $\varepsilon$ as seen from Figs. 4(a) $-4(\mathrm{c})$. This sensitivity to $\varepsilon$ variation reflects the complex shape of $\rho_{L}$ shown in Fig. 2.

For $d_{x y}$-wave case, the line shape of $\rho$ in the bulk has periodic oscillations with the period $0.5 \pi$. The amplitude of the oscillations is reduced with the increase in $\varepsilon$ as shown by the curves B in Figs. 4(d)-4(f). On the other hand, the line shapes of the curves A change drastically with the increase in $\varepsilon$. This sensitivity originates from the complex patterns of $\rho_{L}$ shown in Fig. 3. Although we do not discuss in detail, the line shape of $\rho$ at the N/S interface is also sensitive to a magnetic field $H$ for a fixed $\varepsilon$.

As seen from the above results, by changing the magnitude of the applied magnetic field $H$, the bias voltage $V$, and the rotation angle $\phi$, it is possible to clarify the remarkable deformation of Cooper pairs. Therefore, magnetotunneling 
spectroscopy provides the way to detect bulk symmetry of the pair potential.

We have not explicitly discussed the influence of impurity scattering in $\mathrm{N}$, but one can show that the oscillatory behavior of $\rho$ can be detected if the following two conditions are fulfilled: $l>L$ and $l>L_{0}$, where $l$ is the mean free path in $\mathrm{N}$. To satisfy these conditions, normal metals with high mobility are desirable. Superconducting junctions with 2D-electron gas realized in InAs (Ref. 16) or graphene ${ }^{17}$ are possible candidates due to high electronic mobility in both types of materials. Furthermore, large magnitude of $T_{C}$ or $\Delta_{0}$ helps to satisfy the second condition. From this viewpoint, junctions with high $T_{C}$ cuprates extensively studied by now ${ }^{18}$ are promising candidates. Surface roughness could also lead to a broadening of the oscillatory behavior of $\rho$. This effect is controlled by an effective scattering length within the surface layer (see Refs. 8 and 19). Both bulk and surface scattering lead to mixing of quasiparticle trajectories at different angles, while the general angular shape of the Cooper pair does not change and can be determined at realistic experimental conditions.

In summary, we have studied the Cooper pair shape in normal-metal/superconductor (N/S) junctions by using the quasiclassical Green's function formalism. The quasiparticle interference leads to striking deformations in the shape of a Cooper pair wave function in a normal metal. We also show that the anisotropic shape of Cooper pairs could be resolved by scanning tunneling spectroscopy experiments in magnetic field. The Cooper pair deformation is a common feature of nonuniform superconducting systems in the clean limit. This provides a key concept to explore unknown quantum interference phenomena in superconducting nanostructures.
${ }^{1}$ G. Deutscher and P. G. de Gennes, in Superconductivity, edited by R. D. Parks (Dekker, New York, 1966); B. D. Josephson, in Superconductivity (Ref. 1).

${ }^{2}$ V. L. Berezinskii, JETP Lett. 20, 287 (1974).

${ }^{3}$ M. Sigrist and K. Ueda, Rev. Mod. Phys. 63, 239 (1991).

${ }^{4}$ H. Won, Y. Morita, and K. Maki, Phys. Status Solidi B 244, 4371 (2007); Y. Matsuda, K. Izawa, and I. Vekhter, J. Phys.: Condens. Matter 18, R705 (2006).

${ }^{5}$ F. S. Bergeret, A. F. Volkov, and K. B. Efetov, Rev. Mod. Phys. 77, 1321 (2005).

${ }^{6}$ Y. Tanaka and A. A. Golubov, Phys. Rev. Lett. 98, 037003 (2007); Y. Tanaka, A. A. Golubov, S. Kashiwaya, and M. Ueda, ibid. 99, 037005 (2007); M. Eschrig, T. Lofwander, Th. Champel, J. C. Cuevas, and G. Schon, J. Low Temp. Phys. 147, 457 (2007).

${ }^{7}$ W. Belzig, C. Bruder, and A. L. Fauchere, Phys. Rev. B 58, 14531 (1998).

${ }^{8}$ A. A. Golubov and M. Yu. Kupriyanov, JETP Lett. 67, 501 (1998); 69, 262 (1999).

${ }^{9}$ P. G. de Gennes and D. Saint-James, Phys. Lett. 4, 151 (1963); D. Saint-James, J. Phys. (Paris) 25, 899 (1964).

${ }^{10}$ J. W. Serene and D. Rainer, Phys. Rep. 101, 221 (1983).
${ }^{11}$ M. Eschrig, Phys. Rev. B 61, 9061 (2000).

12 J. M. Rowell and W. L. McMillan, Phys. Rev. Lett. 16, 453 (1966); J. M. Rowell, ibid. 30, 167 (1973).

${ }^{13}$ Y. Tanaka and S. Kashiwaya, Phys. Rev. Lett. 74, 3451 (1995); S. Kashiwaya and Y. Tanaka, Rep. Prog. Phys. 63, 1641 (2000); C. R. Hu, Phys. Rev. Lett. 72, 1526 (1994); C. Bruder, Phys. Rev. B 41, 4017 (1990); Y. Asano, Y. Tanaka, and S. Kashiwaya, ibid. 69, 134501 (2004).

${ }^{14}$ M. Fogelström, D. Rainer, and J. A. Sauls, Phys. Rev. Lett. 79, 281 (1997).

${ }^{15}$ Y. Tanuma, K. Kuroki, Y. Tanaka, R. Arita, S. Kashiwaya, and H. Aoki, Phys. Rev. B 66, 094507 (2002).

${ }^{16}$ H. Takayanagi and T. Akazaki, Phys. Rev. B 52, R8633 (1995).

${ }^{17}$ H. B. Heersche, P. Jarillo-Herrero, J. B. Oostinga1, L. M. K. Vandersypen, and A. F. Morpurgo1, Nature (London) 446, 56 (2007).

${ }^{18}$ M. Covington, M. Aprili, E. Paraoanu, L. H. Greene, F. Xu, J. Zhu, and C. A. Mirkin, Phys. Rev. Lett. 79, 277 (1997); I. Iguchi, W. Wang, M. Yamazaki, Y. Tanaka, and S. Kashiwaya, Phys. Rev. B 62, R6131 (2000).

${ }^{19}$ Yu. S. Barash, A. A. Svidzinsky, and H. Burkhardt, Phys. Rev. B 55, 15282 (1997). 